\title{
Development of a Finite-difference Regularized Solution of the One-Dimensional Inverse Problem of the Wave Process
}

\author{
Abdugany Dzhunusovich Satybaev ${ }^{1}$, Yuliya Vladimirovna Anishchenko ${ }^{1, *}$, \\ Ainagul Zhylkychyevna Kokozova ${ }^{1}$, Aliyma Torozhanovna Mamatkasymova ${ }^{2}$, \\ Guljamal Abdazovna Kaldybaeva ${ }^{3}$ \\ ${ }^{1}$ Department of Information Technology and Management, Faculty of Cybernetics and Information Technology, Osh Technological \\ University, Osh, Kyrgyzstan \\ ${ }^{2}$ Department of Informatics, Naturally-technical Faculty, Osh Technological University, Osh, Kyrgyzstan \\ ${ }^{3}$ Department of Physics, Mathematics and Information Technology, Osh State University, Osh, Kyrgyzstan

\section{Email address:} \\ programm85@gmail.com (Y. V. Anishchenko), abdu-satybaev@mail.ru(A. D. Satybaev), kokozova72@mail.ru(A. Z. Kokozova), \\ mamatkasymova1973@mail.ru(A. T. Mamatkasymova), gkaldybayeva@inbox.ru(G. A. Kaldybaeva) \\ ${ }^{*}$ Corresponding author
}

\section{To cite this article:}

Abdugany Dzhunusovich Satybaev, Yuliya Vladimirovna Anishchenko, Ainagul Zhylkychyevna Kokozova, Aliyma Torozhanovna Mamatkasymova, Guljamal Abdazovna Kaldybaeva. Development of a Finite-difference Regularized Solution of the One-Dimensional Inverse Problem of the Wave Process. American Journal of Applied Mathematics. Vol. 8, No. 2, 2020, pp. 64-73.

doi: $10.11648 /$ j.ajam.20200802.13

Received: March 3, 2020; Accepted: March 23, 2020; Published: April 13, 2020

\begin{abstract}
We consider a one-dimensional inverse problem for a partial differential equation of hyperbolic type with sources the Dirac delta-function and the Heaviside theta-function. The generalized inverse problem is reduced to the inverse problem with data on the characteristics using the method of characteristics and the method of isolation of singularities. At the beginning, the inverse problem of the wave process with data on the characteristics with additional information for the inverse problem without small perturbations is solved by the finite-difference method. Then, for the inverse problem of the wave process with data on the characteristics with additional information with small perturbations, that is, with small changes is used by the finite-difference regularized method, which developed by one of the authors of this article. The convergence of the finite-difference regularized solution to the exact solution of the one-dimensional inverse problem of the wave process on the characteristics is shown, and the theorem on the convergence of the approximate solution to the exact solution is proved. An estimate is obtained for the convergence of the numerical regularized solution to the exact solution, which depends on the grid step, on the perturbations parameter, and on the norm of known functions. From the equivalence of the problems, the one-dimensional inverse problem of the wave process with sources - the Dirac delta-function and the Heaviside theta-function and the one-dimensional inverse problem of the wave process with data on the characteristics, it follows that the solution of the last problem will be the solution of the posed initial problem. An algorithm for solving a finite-difference regularized solution of a generalized one-dimensional inverse problem is constructed.
\end{abstract}

Keywords: One-dimensional Inverse Problem, Wave Process, Dirac Delta-Function, Heaviside Theta-Function, Method of Characteristic, Method of Isolation of Singularities, Finite-Difference Regularized Solution

\section{Introduction}

Inverse problems are the so-called ill-posed problems, the foundations of which were laid by Academicians of the Russian Academy of Sciences Andrei N. Tikhonov [1], Mikhail M. Lavrentiev [2], Corresponding Member of the
Russian Academy of Sciences Valentin K. Ivanov [3].

The inverse problems of wave processes were considered in theoretical terms by the Corresponding members of the Russian Academy of Sciences Vladimir G. Romanov [4], Sergey I. Kabanikhin [5], professor Valery G. Yakhno [6], and they constructed solutions to the posed inverse problems. 
The regularization method for inverse problems was developed by Andrei N. Tikhonov [7], the method of small parameters was constructed by Mikhail M. Lavrentiev [8] and the quasi-solution method - by Valentin K. Ivanov [9]. The projection-difference method for multidimensional inverse problems was developed by Sergey I. Kabanikhin [10], the finite-difference regularization for Volterra integral equation of the first kind was investigated by Abdugany Dzh. Satybaev [11] and a regularization estimate is obtained.

Aliyma T. Mamatkasymova and Abdugany Dzh. Satybaev [12] constructed a finite-difference regularized solution and obtained a convergence estimate for the inverse problem arising in electromagnetic processes.

The purpose of this work is to numerically solve a one-dimensional inverse problem of the wave process proposed by the authors by a finite-difference regularized method, which allows us to construct a numerical algorithm for solving the problem.

\section{Research Overview}

Most of the scientific and technical aspects of the inverse problems of wave propagation in the medium and the mathematical connections between waves and scatterers are determined and presented in work G. Ghavent, G. C. Papanicolaou, P. Sacks, W. Symes [13].

The book, edited by G. Chavent and P. C. Sabatier [14], describes the current state of modeling and the numerical solution of wave propagation and diffraction, their applications, and features of inverse scattering problems on classical and distributed media.

The article set forth by F. Natterer [15] three major methods for solving inverse problems: the method of ray tomography, the method of single-particle emission tomography and the method of positron-emission tomography and described transfer equations transitions near the infrared region to elliptic equations in the diffusion approximation.

In the article by F. Natterer, A. Wiibbeling [16], a numerical calculation of the potential in the Helmholtz equation was laid out and a method was developed which possesses the stability of the solution and the convergence of the solution was shown in the order $\mathrm{O}\left(\mathrm{h}^{4}\right)$.

Modern mathematical modeling of various wave processes (computed tomography, ultrasonic flaw detection, etc.) in the theory of inverse problems and their examples, main features, perspectives are presented in the work of Alexander O. Vatulyan [17].

In the Andrey V. Bayev's dissertation work [18] developed stable methods for solving inverse problems, determined the characteristics of real inhomogeneous layered media, and according to experimental information, clarified the time parameters of the source of disturbances, and also presented practical solutions to a number of actual inverse problems in borehole exploration geophysics.

Dynamic inverse problems, definitions of one or several coefficients of hyperbolic equations or systems, methods for solving one-dimensional inverse problems, scalar inverse problems of wave propagation in layered media, inverse problems for the theory of elasticity and acoustic equations are given in the monograph of Alexander S. Blagoveshchenskii [19].

Alexander V. Avdeev, Vyacheslav I. Priimenko, E. V. Gorbunov, D. V. Zvyagin [20] considered the inverse problem of electromagneto elasticity with electrodynamics of vibrating elastic media.

In the monograph of Sergey I. Kabanikhin [21] outlines methods for studying and solving inverse and ill-posed problems of linear algebra, integral and operator equations, integral geometry, spectral inverse problems and inverse scattering problems; linear ill-posed problems and coefficient inverse problems for hyperbolic, parabolic and elliptic equations were considered; given extensive reference material.

A new globally convergent numerical method is developed for a multidimensional coefficient inverse problem for a hyperbolic PDE with applications in acoustics and electromagnetics. On each iterative step the Dirichlet boundary value problem for a second-order elliptic equation is solved. The global convergence is rigorously established, and numerical experiments are presented in works Larisa Beilina, Michael V. Klibanov [22].

In the book by V. A. Burov and O. D. Rumyantseva [23], inverse wave problems and their applied aspects related to linear and nonlinear acoustic tomography, as well as acoustic thermotomography, are considered. Part I briefly discusses the inverse coherent radiation problems, which are characterized by incorrectness and a strong degree of non-uniqueness. Various approaches to solving inverse wave problems of radiation and incoherent problems of active-passive acoustic thermotomography are described. It is shown that the active-passive mode allows you to determine the set of acoustic and thermal characteristics of the medium within the framework of the general tomographic scheme.

\section{Formulation of the Problem}

Wave processes of natural phenomena (earthquakes and natural explosions, landslides and lavas), electrodynamics and geophysics, etc., are characterized by fields described by second-order partial differential equations:

$$
a(x) \vartheta_{t t}(x, t)+b(x) \vartheta_{t}(x, t)=c(x) \vartheta_{x x}(x, t)+d(x) \vartheta_{x}(x, t)+e(x) \vartheta(x, t),(x, t) \in R_{+}^{2}
$$

where $a(x), b(x), c(x), d(x), e(x)$ are the coefficients of the equation (physical parameters of the equation), $\vartheta(x, t)$ is the function describing the process.

For (1) we consider the initial and boundary conditions of the form:

$$
\left.\vartheta(x, t)\right|_{t<0} \equiv 0,\left.\quad \vartheta_{x}(x, t)\right|_{x=0}=h_{0} \delta(t)+r_{0} \theta(t), t \in R_{+}
$$


here $h_{0}, r_{0}$ are positive constant numbers, $\delta(t)$ is the Dirac delta-function, $\theta(t)$ is the Heaviside theta-function.

Let a time-like form be given with respect to the solution of the direct problem (1)-(2)

$$
\left.\vartheta(x, t)\right|_{x=0}=f(t), t \in[0, T]
$$

Condition (2) means that the process has been at rest up to the time $t=0$ and since the time $t=0$, in connection with the assignment to the boundary $x=0$ of sources with forces $h_{0}$ and $r_{0}$, the physical wave process is starting.

Usually, when considering inverse problems, the correctness (existence, uniqueness, stability) of a solution is established; we established it in the works [24], [25].

A. When coefficients are not equal to zero, the direct problem (1)-(2) is a problem of hyperbolic type (problems: wave equation, acoustics, seismic, electrodynamics, etc.).

B. When the coefficient $a(x)=0$, we have a problem of parabolic type (the problems of: diffusion, thermometry, the distribution of quasi-stationary electromagnetic fields, etc.).

C. When coefficients $a(x)=b(x)=0$ we have a problem of elliptic type (the problems of: gravimetry, geoelectrics of stationary fields, etc.).

The problem (1)-(2) with the following coefficients is transformed:

Problem I. When coefficients $b(x)=d(x)=e(x)=0$ - to the wave equation problem, when $b(x)=0$ - to the oscillations problem;

Problem II. When $a(x)=1, b(x)=e(x)=0, c(x)$ is the wave propagation velocity, $d(x)$ is the density of the medium - to the acoustics problem;

Problem III. When $b(x)=e(x)=0, c(x)$ is the wave propagation velocity, $a(x)=\rho(x)$ is the density of the medium, $d(x)$ is the Lame coefficient — to the seismic problem;

Problem IV. When $a(x)=1, e(x)=d(x)=0, b(x)$ is the electrical conductivity of the medium - to the geoelectric problem;

Problem V. When $a(x)=1, e(x)=d(x)=0, b(x)$ is the electric permeability - to the telegraph equation problem. Problems I, II were investigated by A. Dzh. Satybaev, Problem III - by A. A. Alimkanov, IV - by Yu. V. Anishchenko, V — by A. Zh. Kokozova.

The main problem in solving the inverse problems (1)-(3) is, firstly, the presence of the Dirac delta-function in the boundary condition and secondly, the problem is reduced to a system of nonlinear integral equations [10].

Let in relation to the coefficients of equation a condition is executed

$$
(a(x), b(x), c(x), d(x), e(x)) \in \Lambda_{0}
$$

$$
\left.\begin{array}{l}
\Lambda_{0}=\left\{a(x): a(x) \in C^{6}\left(R_{+}\right), \quad a^{\prime}(+O)=0\right. \\
0<M_{1} \leq a(x) \leq M_{2}, \quad\|a(x)\|_{C^{2}\left(R_{+}\right)} \leq M_{3}
\end{array}\right\}
$$

In condition (4), the smoothness of the function is given an increased one to apply the finite-difference method. Equation (1) is a hyperbolic equation, and from the principle of dependence on the specification of data, the solution to problem (1)-(3) can be considered in the region $\Delta(T)$ [10]:

$$
\Delta(T)=\{(x, t): x \in(0, T),|x|<t<2 T-|x|\}
$$

If condition (4) is met, the additional information is $f(t) \in C^{4}(0, T)$, and the solution to the problem is $\vartheta(x, t) \in(\Delta(T))$.

The inverse problem consists in determining one of the coefficients of the equation (it would be good if all the coefficients of the equation are determined simultaneously, but for these additional conditions must be specified).

\section{The Inverse Problem}

The inverse problem is to determine the function $c(x)$ from problem (1) - (2) when specifying the remaining coefficients of the equation and when specifying additional information about solving the direct problem at the boundary $x=0$, that is, when specifying the function $f(t)$.

The direct problem is to determine the function $\vartheta(x, t)$ when defining the functions $a(x), b(x), c(x), d(x), e(x)$ satisfying condition (3).

\subsection{Methods for Solving the Differential Problem}

\subsubsection{The Method of Characteristic}

We will enter the new variable of

$$
z(x)=\int_{0}^{x} \frac{a(x)}{b(x)} d x
$$

and we will enter the new functions of $b a(z(x))=b(x) / a(x), d a(z(x))=d(x) / a(x)$, $c a(z(x))=c(x) / a(x), e a(z(x))=e(x) / a(x)$, $V(z(x), t)=\vartheta(x, t)$.

We make some calculations:

$$
\begin{aligned}
& \vartheta_{t}(x, t)=V_{t}(z(x), t), \vartheta_{t t}(x, t)=V_{t t}(z(x), t), \\
& \vartheta_{x}(x, t)=V_{z}(z(x), t) \cdot z_{x}^{\prime}(x), \\
& \vartheta_{x x}(x, t)=V_{z z}(z(x), t) \cdot z_{x}^{2}(x)+V_{z}(z(x), t) \cdot z_{x x}(x) .
\end{aligned}
$$

Substituting the last calculations into equation (1), we obtain 


$$
\begin{aligned}
& V_{t t}(z, t)+b a(z) V_{t}(z, t)=V_{z z}(z, t)+c a(z) z_{x x} V_{z}(z, t)+d a(z) z_{x} V_{z}(z, t)+e a(z) V(z, t) \\
& =V_{z z}(z, t)+\left[-\frac{1}{2} \frac{c a_{z}^{\prime}(z)}{c a(z)}+\frac{d a(z)}{\sqrt{c a(z)}}\right] V_{z}(z, t)+e a(z) V(z, t)
\end{aligned}
$$

\subsubsection{The Method of Isolation of Singularities}

We single out the singular and regular parts of the solution of the problem by the method of V. G. Romanov [4], representing the solution of the problem in the form:

$$
V(z, t)=\tilde{V}(z, t)+S(z) \theta(t-|z|)+R(z) \theta_{1}(t-|z|)
$$

where $\widetilde{V}(z, t)$ is a smooth function, $\theta_{1}(t)=t \cdot \theta(t)$.

We make some calculations:

$$
\begin{aligned}
& V_{t}^{\prime}(z, t)=\tilde{V}_{z}(z, t)+S(z) \delta(t-|z|)+R(z) \theta(t-|z|) \\
& V_{t t}(z, t)=\tilde{V}_{z z}(z, t)+S(z) \delta^{\prime}(t-|z|)+R(z) \delta(t-|z|) \\
& V_{z}(z, t)=\tilde{V}_{z}(z, t)+S_{z}^{\prime}(z) \theta(t-|z|)-S(z) \delta(t-|z|)+R_{z}(z) \theta_{1}(t-|z|)-R(z) \theta(t-|z|), \\
& V_{z z}(z, t)=\tilde{V}_{z z}(z, t)+S_{z z}^{\prime \prime}(z) \theta(t-|z|)-2 S_{z}(z) \delta(t-|z|)+S(z) \delta^{\prime}(t-|z|)+ \\
& +R_{z z}^{\prime \prime}(z) \theta_{1}(t-|z|)-2 R_{z}(z) \theta(t-|z|)+R(z) \delta(t-|z|) .
\end{aligned}
$$

Substituting the last calculations into the obtained equation, we obtain

$$
\begin{aligned}
& \tilde{V}_{t t}(z, t)+S(z) \delta^{\prime}(t-|z|)+R(z) \delta(t-|z|)+b a(z)\left[\tilde{V}_{t}(z, t)+S(z) \delta(t-|z|)+R(z) \theta(t-|z|)\right] \\
& =\tilde{V}_{z z}(z, t)+S_{z z}(z) \theta(t-|z|)-2 S_{z}(z) \delta(t-|z|)+S(z) \delta^{\prime}(t-|z|)+R_{z z}(z) \theta_{1}(t-|z|)- \\
& -2 R_{z}(z) \theta(t-|z|)+R(z) \delta(t-|z|)+g a(z) \tilde{V}_{z}(z, t)+g a(z) S_{z}(z) \theta(t-|z|)-g a(z) S(z) \delta(t-|z|)+ \\
& +g a(z) R_{z}(z) \theta_{1}(t-|z|)-g a R(z) \theta(t-|z|)+e a(z)\left[\tilde{V}(z, t)+S(z) \theta(t-|z|)+R(z) \theta_{1}(t-|z|)\right]
\end{aligned}
$$

Here

$$
g a(z)=-\frac{1}{2} \frac{c a_{z}^{\prime}(z)}{c a(z)}+\frac{d a(z)}{\sqrt{c a(z)}}
$$

We collect terms with the same factors $\delta(t-|z|), \theta(t-|z|), \theta_{1}(t-|z|)$ and equate them to zero:

$$
\begin{aligned}
& \delta: 2 S_{z}^{\prime}(z)+[b a(z)+g a(z)] S(z)=0, \\
& \theta: 2 R_{z}^{\prime}(z)+[b a(z)+g a(z)] R(z)-S_{z z}^{\prime \prime}(z)-g a(z) S_{z}^{\prime}(z)-e a(z) S(z)=0, \\
& \theta_{1}: R_{z z}^{\prime \prime}(z)+g a(z) R_{z}^{\prime}(z)+e a(z) R(z)=0 .
\end{aligned}
$$

From the boundary condition (2), we obtain

$$
\left.\vartheta_{x}(x, t)\right|_{x=0}=h_{0} \delta(t)+r_{0} \theta(t)=\left[\left.\left.V_{z}^{\prime}(z, t)\right|_{z=0} \cdot z_{x}^{\prime}\right|_{x=0}\right]=\left[\tilde{V}_{z}^{\prime}(0, t)+S(0) \delta(t)+R(0) \theta(t)\right] \cdot \sqrt{\frac{a(0)}{c(0)}} .
$$

Here $\tilde{V}_{z}^{\prime}(0, t)=0$ is even function. Equating terms at $\delta(t), \theta(t)$, we obtain

$$
\begin{aligned}
& S(0)=\sqrt{\frac{c(0)}{a(0)}} h_{0}=\sqrt{c a(0)} h_{0}, \\
& R(0)=\sqrt{\frac{c(0)}{a(0)}} r_{0}=\sqrt{c a(0)} r_{0} .
\end{aligned}
$$


From the last obtained expressions, we obtain integral equations of the second kind with respect to $S(z), R(z)$ :

$$
\begin{gathered}
S(z)=c a(0) h_{0}-\frac{1}{2} \int_{0}^{z}[b a(\lambda)+g a(\lambda)] S(\lambda) d \lambda, z \in(0, T) \\
R(z)=c a(0) r_{0}-\frac{1}{2} \int_{0}^{z}[b a(\lambda)+g a(\lambda)] R(\lambda) d \lambda+\frac{1}{2} \int_{0}^{z}\left[S_{\lambda \lambda}^{\prime \prime}(\lambda)+g a(\lambda) S_{\lambda}^{\prime}+e a(\lambda) S(\lambda)\right] d \lambda, z \in(0, T)
\end{gathered}
$$

Taking into account that $\left.V(z, t)\right|_{t<0} \equiv 0$, and also from the higher got calculations, we will get a next inverse problem with data on the characteristics:

$$
\begin{gathered}
V_{t t}(z, t)=V_{z z}(z, t)-\left[2 \frac{S_{z}^{\prime}(z)}{S(z)}+b a(z)\right] \cdot V_{z}(z, t)-b a(z) V_{z}(z, t)+e a(z) V(z, t), \quad(z, t) \in \Delta(T) \\
\left.V(z, t)\right|_{t=|z|}=S(z), \quad z \in[0, T] \\
\left.V(z, t)\right|_{z=0}=f(t), \quad t \in[0,2 T]
\end{gathered}
$$

Here the inverse problem is to determine the functions $V(z, t), S(z)$ when known coefficients $b a(z), e a(z)$ (they depend on known functions $b(z), a(z), e(z))$, and with additional information about solving a direct problem (15). If we define the function $S(z)$ from the problem (13)-(15), then by the formula

$$
g a(z)=-2 \frac{S^{\prime}(z)}{S(z)}-b a(z), \quad z \in[0, T]
$$

we define the unknown function $g a(z)$. Using the d'Alembert formula for the direct problem (13) - (14), we obtain a solution of this problem

$$
V(z, t)=[f(t+z)+f(t-z)] / 2+\frac{1}{2} \int_{0}^{t} \int_{t-z+\xi}^{t+z-\xi}\left\{-\left[2 \frac{S_{\xi}^{\prime}(\xi)}{S(\xi)}+b a(\xi)\right] \cdot V_{\xi}(\xi, \tau)-b a(\xi) V_{\tau}(\xi, \tau)+e a(\xi) V(\xi, \tau)\right\} d \tau d \xi(1
$$

When $t=z$, we obtain:

$$
\left.V(z, z)\right|_{t=z}=S(z)=[f(2 z)+f(0)] / 2+\frac{1}{2} \int_{0}^{t} \int_{\xi}^{2 z-\xi}\left\{-\left[2 \frac{S_{\xi}^{\prime}(\xi)}{S(\xi)}+b a(\xi)\right] \cdot V_{\xi}(\xi, \tau)-b a(\xi) V_{\tau}(\xi, \tau)+e a(\xi) V(\xi, \tau)\right\} d \tau d \xi
$$

\subsection{Methods for Solving the Difference Problem}

\subsubsection{Finite-difference Solution}

To solve problem (13) - (15), we enter the grid region

$$
\Delta_{h}(T)=\left\{z_{i}=i h, t_{k}=k h, h=\frac{T}{2 N}, i=\overline{0, N} ; i h \leq k h \leq T-i h\right\} .
$$

where $h$ is the grid step in $z, t$.

The difference analogue of the differential equation (13):

$$
\frac{V_{i}^{k+1}-2 V_{i}^{k}+V_{i}^{k-1}}{h^{2}}=\frac{V_{i+1}^{k}-2 V_{i}^{k}+V_{i-1}^{k}}{h^{2}}-S b a_{i} \frac{V_{i}^{k}-V_{i}^{k-1}}{h}+e a_{i} V_{i}^{k},(i h, k h) \in \Delta h(T)
$$


where

$$
V_{i}^{k}=V(i h, k h), S b a_{i}=2 \frac{S_{i}-S_{i-1}}{h S_{i}}+b a_{i}, b a_{i}=b a(i h), e a_{i}=e a(i h)
$$

From (19) we obtain

$$
V_{i+1}^{k}=V_{i}^{k+1}+V_{i}^{k-1}-V_{i-1}^{k}+S b a_{i} \cdot h\left[V_{i}^{k}-V_{i-1}^{k}\right]++b a_{i} h\left[V_{i}^{k}-V_{i}^{k-1}\right]-e a_{i} h^{2} V_{i}^{k}, i=\overline{1, N-1}, k=\overline{i, N-i}
$$

From the last formula (21) we obtain recurrence formulas:

$$
\begin{aligned}
& V_{i}^{k+1}=V_{i-1}^{k+2}+V_{i-1}^{k}-V_{i-2}^{k+1}+h S b a_{i-1}\left(V_{i-1}^{k+2}-V_{i-2}^{k+1}\right)+h b a_{i-1}\left(V_{i-1}^{k+1}-V_{i-1}^{k}\right)-e a_{i-1} h^{2} V_{i-1}^{k+2}, \\
& i=\overline{2, N-2} ; k=\overline{i-1, N-i-1} \\
& V_{i}^{k-1}=V_{i-1}^{k}+V_{i-1}^{k-2}-V_{i-1}^{k-1}+h S b a_{i-1}\left(V_{i-1}^{k-1}-V_{i-2}^{k-1}\right)+h b a_{i-1}\left(V_{i-1}^{k-1}-V_{i-1}^{k-2}\right)-e a_{i-1} h^{2} V_{i-1}^{k-1}, \\
& i=\overline{2, N-2} ; k=\overline{i-2, N-i-2}
\end{aligned}
$$

Substituting the last recurrence formulas into the right-hand side of formula (21), and then again writing down the recurrence formulas for the next term on the right-hand side and supplying again to the right-hand side of (21), etc. continuing the process, we obtain the difference analogue of the d'Alembert integral formula (17) (that is, the solution of the problem (13) $-(14)$ in the difference form):

$$
\begin{aligned}
& V_{i+1}^{k}=\left(f^{k+i+1}+f^{k-i-1}\right) / 2-h \sum_{p=1}^{i} \sum_{\mu=1}^{p}\left(2 \frac{S_{\mu}-S_{\mu-1}}{h S_{\mu}}+b a_{\mu}\right) \cdot\left(V_{\mu}^{k-i-\mu+2 p}-\right. \\
& \left.-V_{\mu-1}^{k-i-\mu+2 p}\right)+h \sum_{p=1}^{i} \sum_{\mu=1}^{p} b a_{\mu}\left(V_{\mu}^{k-i-\mu+2 p}-V_{\mu}^{k-i-\mu+2 p-1}\right)-h^{2} \sum_{p=1}^{i} \sum_{\mu=1}^{p} e a_{\mu} V_{\mu}^{k-i-\mu+2 p}, \\
& i=\overline{1, N-1} ; k=\overline{i, N-i}
\end{aligned}
$$

In the last formula (22), setting $k=i+1$, we obtain the difference analogue of formula (18)

$$
\begin{aligned}
& S_{i+1}=\left(f^{2 i+2}+f^{0}\right) / 2-h \sum_{p=1}^{i} \sum_{\mu=1}^{p}\left(2 \frac{S_{\mu}-S_{\mu-1}}{h S_{\mu}}+b a_{\mu}\right) \cdot\left(V_{\mu}^{-\mu+2 p+1}-\right. \\
& \left.-V_{\mu-1}^{-\mu+2 p+1}\right)+h \sum_{p=1}^{i} \sum_{\mu=1}^{p} b a_{\mu}\left(V_{\mu}^{-\mu+2 p+1}-V_{\mu}^{-\mu+2 p}\right)-h^{2} \sum_{p=1}^{i} \sum_{\mu=1}^{p} e a_{\mu} V_{\mu}^{-\mu+2 p+1}, i=\overline{1, N-1}
\end{aligned}
$$

Formulas (22) and (23) constitute a system of difference non-linear equations of the second kind.

The difference equations (22) and (23) are written without small values of $O(h)$, then for an exact solution with a small value of $O(h)$ it is possible to obtain the same equations (22), (23) by a small quantity $O(h)$. Let us denote the solution of equations with small quantity $O(h)$ by $\widetilde{V}_{i+1}^{k}, \widetilde{S}_{i+1}$.

Then for the difference of the exact approximate solution of the inverse problem $\left(\widetilde{V}_{i+1}^{k}=\widetilde{V}_{i+1}^{k}-V_{i+1}^{k}, \widetilde{S}_{i+1}=\widetilde{S}_{i+1}-S_{i+1}\right)$, we obtain the following expressions:

$$
\begin{aligned}
& \bar{V}_{i+1}^{k}=-h \sum_{p=1}^{i} \sum_{\mu=1}^{p}\left\{b a_{\mu}\left(\bar{V}_{\mu}^{k-i-\mu+2 p}-\bar{V}_{\mu-1}^{k-i-\mu+2 p}\right)+\left(2 \frac{\bar{S}_{\mu}-\bar{S}_{\mu-1}}{h \bar{S}_{\mu}}\right)\left(V_{\mu}^{k-i-\mu+2 p}-V_{\mu-1}^{k-i-\mu+2 p}\right)+\right. \\
& \left.+\left(2 \frac{\tilde{S}_{\mu}-\tilde{S}_{\mu-1}}{h \tilde{S}_{\mu}}\right)\left(\bar{V}_{\mu}^{k-i-\mu+2 p}-\bar{V}_{\mu-1}^{k-i-\mu+2 p}\right)\right\}+h \sum_{p=1}^{i} \sum_{\mu=1}^{p} b a_{\mu}\left(\bar{V}_{\mu}^{k-i-\mu+2 p}-\bar{V}_{\mu}^{k-i-\mu+2 p-1}\right)- \\
& -h^{2} \sum_{p=1}^{i} \sum_{\mu=1}^{p} e a_{\mu} \bar{V}_{\mu}^{k-i-\mu+2 p}+O(h), i=\overline{1, N-1} ; k=\overline{i, N-i}
\end{aligned}
$$




$$
\begin{aligned}
& \bar{S}_{i+1}=-h \sum_{p=1}^{i} \sum_{\mu=1}^{p}\left\{b a_{\mu}\left(\bar{V}_{\mu}^{-\mu+2 p+1}-\bar{V}_{\mu-1}^{-\mu+2 p+1}\right)+\left(2 \frac{\bar{S}_{\mu}-\bar{S}_{\mu-1}}{h \underline{S}_{\mu}}\right)\left(V_{\mu}^{-\mu+2 p+1}-V_{\mu-1}^{-\mu+2 p+1}\right)+\right. \\
& \left.+\left(2 \frac{\tilde{S}_{\mu}-\tilde{S}_{\mu-1}}{h \tilde{S}_{\mu}}\right)\left(\bar{V}_{\mu}^{-\mu+2 p+1}-\bar{V}_{\mu-1}^{-\mu+2 p+1}\right)\right\}+h \sum_{p=1}^{i} \sum_{\mu=1}^{p} b a_{\mu}\left(\bar{V}_{\mu}^{-\mu+2 p+1}-\bar{V}_{\mu}^{-\mu+2 p}\right)- \\
& -h^{2} \sum_{p=1}^{i} \sum_{\mu=1}^{p} e a_{\mu} \bar{V}_{\mu}^{-\mu+2 p+1}+O(h), i=\overline{1, N-1}
\end{aligned}
$$

To estimate (24), (25), we enter the notation

$$
\begin{aligned}
& B A=\max _{i=\overline{0, N}}\left|b a_{i}\right|, \quad E A=\max _{i=0, N}\left|e a_{i}\right|, \\
& \bar{Z}_{i}=\max _{k=i, 2 N-i}\left|\bar{V}_{i}^{k}\right|, \quad i=\overline{0, N} ; \\
& \bar{S}_{i}=\max _{i=\overline{0, N}}\left|S_{i}\right|, \quad \underline{S_{i}}=\min _{i=0, N}\left|S_{i}\right|
\end{aligned}
$$

We estimate the expressions (24), (25)

$$
\begin{aligned}
& \bar{Z}_{i+1} \leq 2 h N \cdot B A \sum_{p=1}^{i} \bar{Z}_{p}+h 8 N \sum_{p=1}^{i} \frac{\bar{S}_{p}}{h \underline{S}_{p}} V_{p}+h 8 N \sum_{p=1}^{i} \frac{\bar{S}_{p}}{h \underline{S}_{p}} \bar{V}_{p}+2 h N \cdot B A \sum_{p=1}^{i} \bar{Z}_{p}+h^{2} N \times \\
& \times E A \sum_{p=1}^{i} \bar{Z}_{p}+O(h)=2 T \cdot B A \sum_{p=1}^{i} \bar{Z}_{p}+16 N \sum_{p=1}^{i} \overline{\bar{S}}_{p} \bar{Z}_{p}+2 T \cdot B A \sum_{p=1}^{i} Z_{p}+T \cdot E A \cdot h \sum_{p=1}^{i} \bar{Z}_{p}+O(h), \quad i=\overline{1, N-1} \\
& \bar{S}_{i+1} \leq 2 h N \cdot B A \sum_{p=1}^{i} \bar{Z}_{p}+h 8 N \sum_{p=1}^{i} \frac{\bar{S}_{p}}{h \underline{S}_{p}} V_{p}+h 8 N \sum_{p=1}^{i} \frac{\bar{S}_{p}}{h \underline{S}_{p}} \bar{V}_{p}+2 h N \cdot B A \sum_{p=1}^{i} \bar{Z}_{p}+h^{2} N \times \\
& \quad \times E A \sum_{p=1}^{i} \bar{Z}_{p}+O(h)=2 T \cdot B A \sum_{p=1}^{i} \bar{Z}_{p}+16 N \sum_{p=1}^{i} \overline{\bar{S}}_{p} \bar{Z}_{p}+2 T \cdot B A \sum_{p=1}^{i} \bar{Z}_{p}+T \cdot E A \cdot h \sum_{p=1}^{i} \bar{Z}_{p}+O(h), i=\overline{1, N-1}
\end{aligned}
$$

Here $\overline{\bar{S}}_{p}=\frac{\bar{S}_{p}}{\underline{S}_{p}}$, if we denote by $Z_{i+1}=\max _{i=0, N-1}\left(\bar{Z}_{i+1}, \overline{\bar{S}}_{i+1}\right)$, then

$$
Z_{i+1} \leq 4 T \cdot B A \sum_{p=1}^{i} Z_{p}+16 N S \sum_{p=1}^{i} Z_{p}+T \cdot E A \cdot h \sum_{p=1}^{i} Z_{p}+O(h)
$$

Using the discrete analogue of the Gronwall - Bellman lemma from the last estimate, we obtain

$$
Z_{i+1}^{O(h)}=O(h) \cdot[\exp (4 T \cdot B A+16 N S+h T \cdot E A)]
$$

Theorem 4.1. The conditions (4) and (6), (solution $V(z, t) \in C^{4}(\Delta(T))$ ) are fulfilled, then the finite-difference solution $\left(V_{i}^{k}, S_{i}\right.$ ) of the inverse problem (13)-(15) constructed converges to the exact solution $\left(\widetilde{V}_{i}^{k}, \widetilde{S}_{i}\right)$ of the inverse problem with a speed of order $O(h)$ and has the estimate (30).

\subsubsection{Finite-difference Regularized Solution}

Let the additional information for the inverse problem be given with an error of $\mathcal{E}$, that is satisfied:

$$
\left|f(t)-f^{\varepsilon}(t)\right|<\varepsilon, \varepsilon \text { is a small number }
$$


Then for a finite-difference regularized solution of the inverse problem (we denote it by $\hat{V}_{i+1}^{k, \varepsilon}, \hat{S}_{i+1}^{\varepsilon}$, we can also obtain formulas of the form (22) and (23):

$$
\begin{gathered}
\hat{V}_{i+1}^{k, \varepsilon}=\left(f^{k+i+1, \varepsilon}+f^{k-i-1, \varepsilon}\right) / 2-h \sum_{p=1}^{i} \sum_{\mu=1}^{p}\left(2 \frac{\hat{S}_{\mu}^{\varepsilon}-\hat{S}_{\mu-1}^{\varepsilon}}{h S_{\mu}}+b a_{\mu}\right) \cdot\left(\hat{V}_{\mu}^{k-i-\mu+2 p, \varepsilon}-\right. \\
\left.-\hat{V}_{\mu-1}^{k-i-\mu+2 p, \varepsilon}\right)+h \sum_{p=1}^{i} \sum_{\mu=1}^{p} b a_{\mu}\left(\hat{V}_{\mu}^{k-i-\mu+2 p, \varepsilon}-\hat{V}_{\mu}^{k-i-\mu+2 p-1, \varepsilon}\right)-h^{2} \sum_{p=1}^{i} \sum_{\mu=1}^{p} e a_{\mu} \hat{V}_{\mu}^{k-i-\mu+2 p, \varepsilon}, i=\overline{1, N-1} ; k=\overline{i, N-i} \\
\hat{S}_{i+1}^{\varepsilon}=\left(f^{2 i+2, \varepsilon}+f^{0, \varepsilon}\right) / 2-h \sum_{p=1}^{i} \sum_{\mu=1}^{p}\left(2 \frac{\hat{S}_{\mu}^{\varepsilon}-\hat{S}_{\mu-1}^{\varepsilon}}{h S_{\mu}}+b a_{\mu}\right) \cdot\left(\hat{V}_{\mu}^{-\mu+2 p+1, \varepsilon}-\right. \\
\left.-\hat{V}_{\mu-1}^{-\mu+2 p+1, \varepsilon}\right)+h \sum_{p=1}^{i} \sum_{\mu=1}^{p} b a_{\mu}\left(\hat{V}_{\mu}^{-\mu+2 p+1, \varepsilon}-\hat{V}_{\mu}^{-\mu+2 p, \varepsilon}\right)-h^{2} \sum_{p=1}^{i} \sum_{\mu=1}^{p} e a_{\mu} \hat{V}_{\mu}^{-\mu+2 p+1, \varepsilon}, i=\overline{1, N-1}
\end{gathered}
$$

Taking now from formulas (22), (23) of formulas (32), (33), we obtain

$$
\begin{aligned}
& \breve{V}_{i+1}^{k}= V_{i+1}^{k}-\hat{V}_{i+1}^{k, \varepsilon}=\left(f^{k+i+1}-f^{k+i+1, \varepsilon}+f^{k-i-1, \varepsilon}-f^{k-i-1}\right) / 2-h \sum_{p=1}^{i} \sum_{\mu=1}^{p}\left\{b a_{\mu}\left(\hat{V}_{\mu}^{k-i-\mu+2 p}-\hat{V}_{\mu-1}^{k-i-\mu+2 p}\right)+\right. \\
&\left.+\left(2 \frac{\hat{S}_{\mu}-\hat{S}_{\mu-1}}{h \breve{S}_{\mu}}\right)\left(V_{\mu}^{k-i-\mu+2 p, \varepsilon}-V_{\mu-1}^{k-i-\mu+2 p, \varepsilon}\right)+\left(2 \frac{S_{\mu}-S_{\mu-1}}{h S_{\mu}}\right)\left(\hat{V}_{\mu}^{k-i-\mu+2 p}-\hat{V}_{\mu-1}^{k-i-\mu+2 p}\right)\right\}+ \\
&+h \sum_{p=1}^{i} \sum_{\mu=1}^{p} b a_{\mu}\left(\hat{V}_{\mu}^{k-i-\mu+2 p}-\hat{V}_{\mu}^{k-i-\mu+2 p-1}\right)-h^{2} \sum_{p=1}^{i} \sum_{\mu=1}^{p} e a_{\mu} \hat{V}_{\mu}^{k-i-\mu+2 p}, i=\overline{1, N-1} ; k=\overline{i, N-i} \\
& \breve{S}_{i+1}=S_{i+1}-\hat{S}_{i+1}^{\varepsilon}=\left(f^{2 i+2}-f^{2 i+2, \varepsilon}+f^{0, \varepsilon}-f^{0}\right) / 2-h \sum_{p=1}^{i} \sum_{\mu=1}^{p}\left\{b a_{\mu}\left(\hat{V}_{\mu}^{-\mu+2 p+1}-\hat{V}_{\mu-1}^{-\mu+2 p+1}\right)+\right. \\
&+\left(2 \frac{\left.\left.\hat{S}_{\mu}-\hat{S}_{\mu-1}\right)\left(V_{\mu}^{-\mu+2 p+1, \varepsilon}-V_{\mu-1}^{-\mu+2 p+1, \varepsilon}\right)+\left(2 \frac{S_{\mu}-S_{\mu-1}}{h S_{\mu}}\right)\left(\hat{V}_{\mu}^{-\mu+2 p+1}-\hat{V}_{\mu-1}^{-\mu+2 p+1}\right)\right\}+}{\left.h \hat{S}_{\mu}\right)}\right. \\
&+h \sum_{p=1}^{i} \sum_{\mu=1}^{p} b a_{\mu}\left(\hat{V}_{\mu}^{-\mu+2 p+1}-\hat{V}_{\mu}^{-\mu+2 p}\right)-h^{2} \sum_{p=1}^{i} \sum_{\mu=1}^{p} e a_{\mu} \hat{V}_{\mu}^{-\mu+2 p+1}, i=\overline{1, N-1}
\end{aligned}
$$

Taking into account the introduced notation (26), we estimate the last equations:

$$
\begin{array}{cc}
\breve{Z}_{i+1} \leq \varepsilon+2 T \cdot B A \sum_{p=1}^{i} \breve{Z}_{p}+16 N \sum_{p=1}^{i} \overline{\bar{S}}_{p} \breve{Z}_{p}+2 T \cdot B A \sum_{p=1}^{i} Z_{p}+T \cdot E A \cdot h \sum_{p=1}^{i} \breve{Z}_{p}, & i=\overline{1, N-1} \\
\breve{S} \leq \varepsilon+h T \cdot B A \sum_{p=1}^{i} \breve{Z}_{p}+16 N \sum_{p=1}^{i} \overline{\bar{S}}_{p} \breve{Z}_{p}+2 T \cdot B A \sum_{p=1}^{i} Z_{p}+T \cdot E A \cdot h \sum_{p=1}^{i} \breve{Z}_{p}, \quad i=\overline{1, N-1}
\end{array}
$$

Denote by $Z_{i+1}^{\delta}=\max _{i=0, N-1}\left\{\breve{Z}_{i+1}, \breve{S}_{i+1}\right\}$, then from the last expressions we get:

$$
Z_{i+1}^{\varepsilon} \leq \varepsilon+4 T \cdot B A \sum_{p=1}^{i} Z_{p}^{\varepsilon}+16 N S \sum_{p=1}^{i} Z_{p}^{\varepsilon}+T \cdot E A \cdot h \sum_{p=1}^{i} Z_{p}^{\varepsilon}, \quad i=\overline{1, N-1}
$$

Using the Gronwall - Bellman inequality, we obtain 


$$
Z_{i+1}^{z}=\delta \cdot \exp [4 T \cdot B A+16 N S+h T \cdot B A]
$$

Then the estimate of a finite-difference regularized solution of the inverse problem has the form:

$$
Z_{i+1}^{z, O(h)}=(\varepsilon+O(h)) \delta \exp (4 T \cdot B A+16 N S+h T \cdot E A)
$$

Theorem 4.2. Suppose that conditions (4), (6) and (31) are satisfied, then the constructed finite-difference regularized solution converges to the exact solution of the inverse problem with a speed of order $O(h)$ and has the estimate (40).

\subsubsection{The Algorithm for Obtaining a Finite-Difference Regularized Solution of the Generalized One-Dimensional Inverse Problem (1)-(3)}

Thus, the finite-difference regularized solution of the inverse problem (13) - (15) is $S_{i}^{\varepsilon, O(h)}=V_{i}^{i, \varepsilon, O(h)}$, then by formula (16) we have:

$$
g a_{i}^{\varepsilon, O(h)}=-2 \frac{S_{i}^{\varepsilon, O(h)}-S_{i-1}^{\varepsilon, O(h)}}{h S_{i}^{\varepsilon, O(h)}}-b a_{i}, \quad i=\overline{0, N}
$$

We note that it is possible to obtain from the formula

$$
c a_{z}^{\prime}(z)=2 d a(z) \cdot \sqrt{c a(z)}-2 g a(z) c a(z)
$$

we integrate

$$
c a(z)=c a(0)+2 \int_{0}^{z}[\sqrt{c a(\lambda)} d a(\lambda)-c a(\lambda) \cdot g a(\lambda)] d \lambda
$$

Using the quadrature formulas for the integral, we obtain the solution of the inverse problem (7), (14), (15):

$$
c a_{i}^{\varepsilon, O(h)}=c a(0)+2 \sum_{p=1}^{i}\left[\sqrt{c a_{i}^{\varepsilon, O(h)}} d a_{i}-c a_{i}^{\varepsilon, O(h)} g a_{i}^{\varepsilon, O(h)}\right] \cdot h, i=\overline{1, N}
$$

Having determined $c a_{i}^{\varepsilon, O(h)}$ we can get:

$$
c_{i}^{\varepsilon, O(h)}=c a_{i}^{\varepsilon, O(h)} \cdot a_{i}, i=\overline{0, N}
$$

$c_{i}^{\varepsilon, O(h)}$ is a finite-difference regularized solution of the equivalent inverse problem (1) - (3).

Comment. Other coefficients of equation (1) can also be recovered by the same method and obtain their finite-difference regularized solutions.

\section{Conclusion}

This article investigates the inverse problem of the wave process with boundary data of the Dirac delta-function and the Heaviside theta-function. The problem is reduced to a problem with data on characteristics using the method of characteristics and the method of isolation of singularities. To the last problem with the data on the characteristics is applied the finite-difference method, and to relatively small changes with additional information is applied the finite-difference regularized method, which developed by A. Dzh. Satybaev. For numerical implementation constructed an algorithm for solving the problem.

\section{References}

[1] A. N. Tikhonov and V. Ya. Arsenin, Methods for solving ill-posed problems, Moscow, The science, 1979 (in Russian).

[2] M. M. Lavrentiev, V. G. Romanov and S. P. Shishatsky, Ill-posed problems of mathematical physics and analysis, Moscow, The science, 1980 (in Russian).

[3] V. K. Ivanov, V. V. Vasin and V. P. Tanana, The theory of linear ill-posed problems and its applications, Moscow, The science, 1978 (in Russian).

[4] V. G. Romanov, The stability in inverse problems, Moscow, The scientific world, 2005 (in Russian).

[5] S. I. Kabanikhin, Inverse and ill-posed problems, Novosibirsk, Siberian Scientific Publishing House, 2009 (in Russian). 
[6] V. G. Yakhno, Inverse problems for differential equations of elasticity, Moscow, The science, Siberian Branch, 1990 (in Russian).

[7] A. N. Tikhonov, Regularization of ill-posed problems, in: Reports of the Academy of Sciences of the USSR, Vol. 153, No. 1, (1963), 49-52 (in Russian).

[8] M. M. Lavrentiev, About of the first kind integral equations, in: Reports of the Academy of Sciences of the USSR, Vol. 127, No. 1, (1959) (in Russian).

[9] V. K. Ivanov, About ill-posed problems, in: Mathematical collection, Vol. 61, No. 2, (1963) (in Russian).

[10] S. I. Kabanikhin, Projection-difference methods for determining the coefficients of hyperbolic equations, Novosibirsk, Nauka, 1988 (in Russian).

[11] A. Dzh. Satybaev, Finite-difference regularization of the first-kind integral Volterra equation, Investigations on integro-differential equations, Frunze, Ilim, Vol. 18, (1985), 196-198 (in Russian).

[12] A. T. Mamatkasymova and A. Dzh. Satybaev, Development of a finite-difference regularized solution of the one-dimensional inverse problem arising in electromagnetic processes, XXXVII International Scientific and Practical Conference "Natural and Mathematical Sciences in the Modern World", Collection of articles, No. 1 (37), Novosibirsk, SibAC, (2016) (in Russian).

[13] G. Chavent, G. C. Papanicolaou, P. Sacks and W. Symes, Inverse Problems in Wave Propogation, 1997 (in English).

[14] G. Chavent and P. C. Sabatier, Inverse Problems of Wave Propagation and Diffraction, Springer, 1997. - 377 p. (in English).

[15] F. Natterer, Imaging and Inverse Problems of Partial Differential Equations, https://www.researchgate.net/publication/228791970_Imaging and inverse problems of partial differential equations [accessed Dec 30 2018] (in English).

[16] F. Natterer and F. Wiibbeling, A Finite Difference Method for the Inverse Scattering Problem at Fixed Frequency. Inverse Problems in Mathematical Physics in: Proceedings of The Lapaland Conference on Inverse Problems Held at Saariselkä, Finland, 14-20 June 1992. Editors: Lassi Päivärinta, Erkki Somersalo (in English).
[17] A. O. Vatulyan, Mathematical models and inverse problems. Mathematics, Rostovon-Don, Rostov State University, 1998 (in Russian).

[18] A. V. Bayev, Inverse problems of wave propagation in inhomogeneous layered media and methods for their solution, Abstract of dissertation for the degree of Doctor of Physical and Mathematical Sciences, Moscow State University named after M. V. Lomonosov, Faculty of Computational Mathematics and Cybernetics, Moscow, 1997 (in Russian).

[19] A. S. Blagoveshchenskii, Inverse Problems of Wave Processes in: Inverse and Ill-Posed Problems Series 23 (in English).

[20] A. V. Avdeev, V. I. Priimenko, E. V. Gorbunov and D. V. Zvyagin, Direct and Inverse Problems of Electromagnetoelasticity in: 5th International Congress of the Brazilian Geophysical Society, SaoPaulo, Brazil, Sept 28-Oct 2, 1997, 658-660 (in English).

[21] S. I. Kabanikhin, Inverse and ill-posed problems: theory and applications, Berlin; Boston: De Gruyter, - Inverse and ill-posed problems series, 1381-4524; 55, 459 p. (in English).

[22] Larisa Beilina and Michael V Klibanov, A globally convergent numerical method for a coefficient inverse problem, SIAM Journal on Scientific Computing: Society for Industrial and Applied Mathematics. Vol. 31, N1. 2008/10/16. 478-509 (in English).

[23] V. A. Burov, O. D. Rumyantseva, Inverse wave problems of acoustic tomography. Part I: Inverse problems of radiation in acoustics. (Ed. Stereotype). - Moscow: Moscow, (2018). - 384 p. (in Russian).

[24] A. Dzh. Satybaev, Yu. V. Anishchenko, A. Zh. Kokozova, A. A. Alimkanov, The uniqueness of the solution of the two-dimensional direct problem of a wave process with an instantaneous source and a flat boundary. AIP Conference Proceedings 1997, 020063 (2018); 10.1063/1.5049057 (in English).

[25] A. Dzh. Satybaev, A. Zh. Kokozova, Yu. V. Anishchenko, A. A. Alimkanov, Numerical solution of a two-dimensional direct problem of the wave process. AIP Conference Proceedings 1997, 020045 (2018); 10.1063/1.5049039 (in English). 